\title{
Radial peripapillary capillary density in superior segmental optic hypoplasia measured with OCT angiography
}

\author{
Maiko Abe ${ }^{1}$, Kazuko Omodaka ${ }^{1,2}$, Tsutomu Kikawa ${ }^{3}$ and Toru Nakazawa ${ }^{1,2^{*}}$
}

\begin{abstract}
Background: To investigate the diagnostic power of radial peripapillary capillary (RPC) density, measured with optical coherence tomography angiography (OCT-A), in patients with superior segmental optic hypoplasia (SSOH).

Methods: Forty subjects with $\mathrm{SSOH}$ and 40 age- and axial length-matched control subjects were retrospectively registered for this study. $\mathrm{SSOH}$ was defined as intraocular pressure less than $21 \mathrm{mmHg}$ with the presence of two of the following: superior rim thinning, superior entrance of the central retinal artery, scleral halo, and pale optic disc; as well as non-progressive visual field loss. RPC density was measured with swept-source OCT-A (Triton, Topcon) overall, in the quadrants, and in the 12 clock-wise sectors. Changes in RPC density were also compared in $\mathrm{SSOH}$ patients and age-matched patients with mild- or moderate-stage of glaucoma. RPC density was compared in pairs of groups with Welch's t-test. Diagnostic power was assessed with the area under the receiver operating characteristics curve (AUC).
\end{abstract}

Results: Overall cpRNFLT was significantly different in the normal $(106.7 \pm 9.5 \mu \mathrm{m})$ and SSOH $(77.2 \pm 13.7 \mu \mathrm{m}$, $p<0.001)$ subjects. RPC density overall and in the superior, nasal, and inferior quadrants was significantly lower in the SSOH group (all, $p<0.001$ ), but not in the temporal $(p=0.756)$ quadrant. The diagnostic power of RPC density was highest in the superior quadrant (AUC $=0.928$ ) and the 1 o'clock sector $(0.896)$. Comparing the $\mathrm{SSOH}$ and glaucoma patients showed that there were no significant differences in RPC density either overall $(p=0.391)$ or in the superior quadrant $(p=0.268)$, while RPC density was significantly higher in the inferior $(p=0.005)$ and temporal quadrants $(p<0.001)$ and lower in the nasal quadrant $(p=0.029)$.

Conclusions: Low RPC density was found in the three non-temporal quadrants of the optic nerve head in $\mathrm{SSOH}$ patients, in comparison to normal subjects. Regionally, RPC density in $\mathrm{SSOH}$ was lower in the nasal quadrant and higher in the inferior and temporal quadrants in comparison to glaucoma patients. Measuring RPC density with OCT-A may help the diagnosis of $\mathrm{SSOH}$ and may improve the management of glaucoma.

Keywords: Optical coherence tomography angiography, Diagnosis, Radial peripapillary capillary density, Superior segmental optic hypoplasia

\footnotetext{
* Correspondence: ntoru@oph.med.tohoku.ac.jp

'Department of Ophthalmology, Tohoku University Graduate School of Medicine, Sendai, Japan

${ }^{2}$ Department of Ophthalmic Imaging and Information Analytics, Tohoku University Graduate School of Medicine, Sendai, Japan

Full list of author information is available at the end of the article
}

(c) The Author(s). 2020 Open Access This article is licensed under a Creative Commons Attribution 4.0 International License, which permits use, sharing, adaptation, distribution and reproduction in any medium or format, as long as you give appropriate credit to the original author(s) and the source, provide a link to the Creative Commons licence, and indicate if changes were made. The images or other third party material in this article are included in the article's Creative Commons licence, unless indicated otherwise in a credit line to the material. If material is not included in the article's Creative Commons licence and your intended use is not permitted by statutory regulation or exceeds the permitted use, you will need to obtain permission directly from the copyright holder. To view a copy of this licence, visit http://creativecommons.org/licenses/by/4.0/ The Creative Commons Public Domain Dedication waiver (http://creativecommons.org/publicdomain/zero/1.0/) applies to the data made available in this article, unless otherwise stated in a credit line to the data. 


\section{Background}

Superior segmental optic hypoplasia $(\mathrm{SSOH})$ is a congenital anomaly of the optic nerve head $(\mathrm{ONH})$ characterized by superior entry of the central retinal vessels, superior peripapillary scleral halo, and reduced circumpapillary retinal nerve fiber layer thickness (cpRNFLT) with non-progressive inferior-sector visual field defects. In Asia, normal-tension glaucoma (NTG) is the primary subtype of open-angle glaucoma [1] making it important to differentiate SSOH from NTG [2]. Previously, it was reported that $\mathrm{SSOH}$ could be identified based on the location of cpRNFLT loss [3] and structural abnormalities in the extension of the retinal pigment epithelium (RPE) over the nasal disc margin [4], both measured with spectral-domain optical coherence tomography (OCT). Thus, both fundus photography and OCT may be useful in the clinical treatment of $\mathrm{SSOH}$.

We previously showed that tissue mean blur rate (MT), representing blood flow (BF) in the $\mathrm{ONH}$, decreases in $\mathrm{SSOH}$ [5]. MT is also correlated with deep$\mathrm{ONH} \mathrm{BF}$, but not surface-ONH BF [6]. Recently, OCT angiography (OCT-A), based on swept-source OCT, has become available clinically and is increasingly important for visualizing vessels both in the retina and $\mathrm{ONH}$. The density of radial peripapillary capillaries (RPCs), a network of surface nerve fiber layers, has been shown to have diagnostic power for glaucoma [7]. However, until now, there have been no reports on changes in RPC density or the diagnostic power of RPC density in SSOH. This study is thus the first to assess the microvasculature of the peripapillary retina and investigate whether vascular parameters are useful for diagnosing $\mathrm{SSOH}$.

\section{Methods}

Eighty eyes of 40 normal and $40 \mathrm{SSOH}$ patients were included. $\mathrm{SSOH}$ was diagnosed by glaucoma specialists after a careful differential diagnosis for glaucoma [4]. $\mathrm{SSOH}$ diagnosis was based on the presence of more than two of the following four symptoms: superior rim thinning, superior entrance of the central retinal artery, scleral halo, and pale optic disc; combined with nonprogressive visual field loss (in an average of $6.3 \pm 4.8$ visual field tests), peripheral visual field defects in the $\mathrm{V}$ 4 target of the Goldmann visual field test, and intraocular pressure (IOP) less than $21 \mathrm{mmHg}$. Mean deviation measurements used reliable data from Humphrey field analyzer standard automated perimetry. We also recruited 50 separate patients, including $27 \mathrm{SSOH}$ patients and 23 age-matched mild- or moderate-stage glaucoma patients, to compare regional differences in RPC density. RPC density was measured with OCT-A (Triton, Topcon), as previously described [7], overall, in the superior, temporal, inferior, and nasal quadrants, and in 12 clockwise sectors. Swept-source OCT (SS-OCT) uses a tissue- penetrating laser system with a long central wavelength of $1050 \mathrm{~nm}$ and allows patients to easily maintain good fixation during scanning due to its low glare. The device is also equipped with an eye-tracking system. Thus, OCT-A with SS-OCT has led to significant improvements in the observation of retinal capillaries, both at the surface and in the deep areas of the $\mathrm{ONH}$, in glaucoma patients. cpRNFLT was measured with sweptsource OCT.

This study adhered to the tenets of the Declaration of Helsinki, and the protocols were approved by the institutional review board of the Tohoku University Graduate School of Medicine (study 2017-1-290). Clinical findings and other characteristics, including age, sex, axial length, and IOP, were compared in pairs of groups with Welch's t-test. The area under the receiver operating characteristic curve (AUC) was calculated for differentiating normal subjects and SSOH patients based on OCT-A parameters. All statistical analyses were performed with JMP software (Pro version 13.1.0, SAS Institute Japan Inc., Tokyo, Japan).

\section{Results}

There were no differences between the groups in age (normal: $39.5 \pm 8.2$, SSOH: $41.2 \pm 18.0, p=0.326$ ), axial length $(24.6 \pm 0.8,25.1 \pm 1.3, p=0.088)$, or IOP $(14.0 \pm$ $2.2,14.1 \pm 2.5, p=0.749$ ). Figure 1 shows a representative $\mathrm{SSOH}$ patient without visual field progression over 5 years and a normal subject; RPC density in the superior to nasal quadrants is clearly lower in the $\mathrm{SSOH}$ subject. Table 1 shows differences in RPC density between the normal and SSOH groups. There were significant differences in overall RPC density and in the superior, nasal, and inferior (all: $p<0.001)$, but not temporal $(p=0.756)$ quadrants. RPC density was significantly lower in the 1 , $2,4,5,7,9,11$, and 12 o'clock sectors. Table 2 shows the diagnostic power of RPC density. The highest AUC was in the superior quadrant (AUC $=0.928$, Fig. 1e), with a cutoff value of $42.14 \%$ (83\% sensitivity and $88 \%$ specificity). The 1 o'clock sector had the highest AUC (0.896, Fig. 1f), with a cutoff value of $34.8 \%$ ( $80 \%$ sensitivity and $83 \%$ specificity).

We also compared changes in RPC density in 27 $\mathrm{SSOH}$ patients and 23 age-matched patients with mildor moderate-stage of glaucoma. There were no differences between the groups in age (normal: $39.5 \pm 8.2$, SSOH: $41.2 \pm 18.0, p=0.326)$, IOP $(14.0 \pm 2.2,14.1 \pm 2.5$, $p=0.749)$ or axial length $(24.6 \pm 0.8,25.1 \pm 1.3, p=$ $0.088)$, but there was a significant difference in mean deviation $(24.6 \pm 0.8,25.1 \pm 1.3, p=0.088)$. We found that $\mathrm{RPC}$ density in the $\mathrm{SSOH}$ patients was significantly higher in the inferior $(p=0.005,0.725)$ and temporal $(p<0.001,0.781)$ quadrants and lower in the nasal quadrant $(p=0.029,0.688)$. There was no difference in 

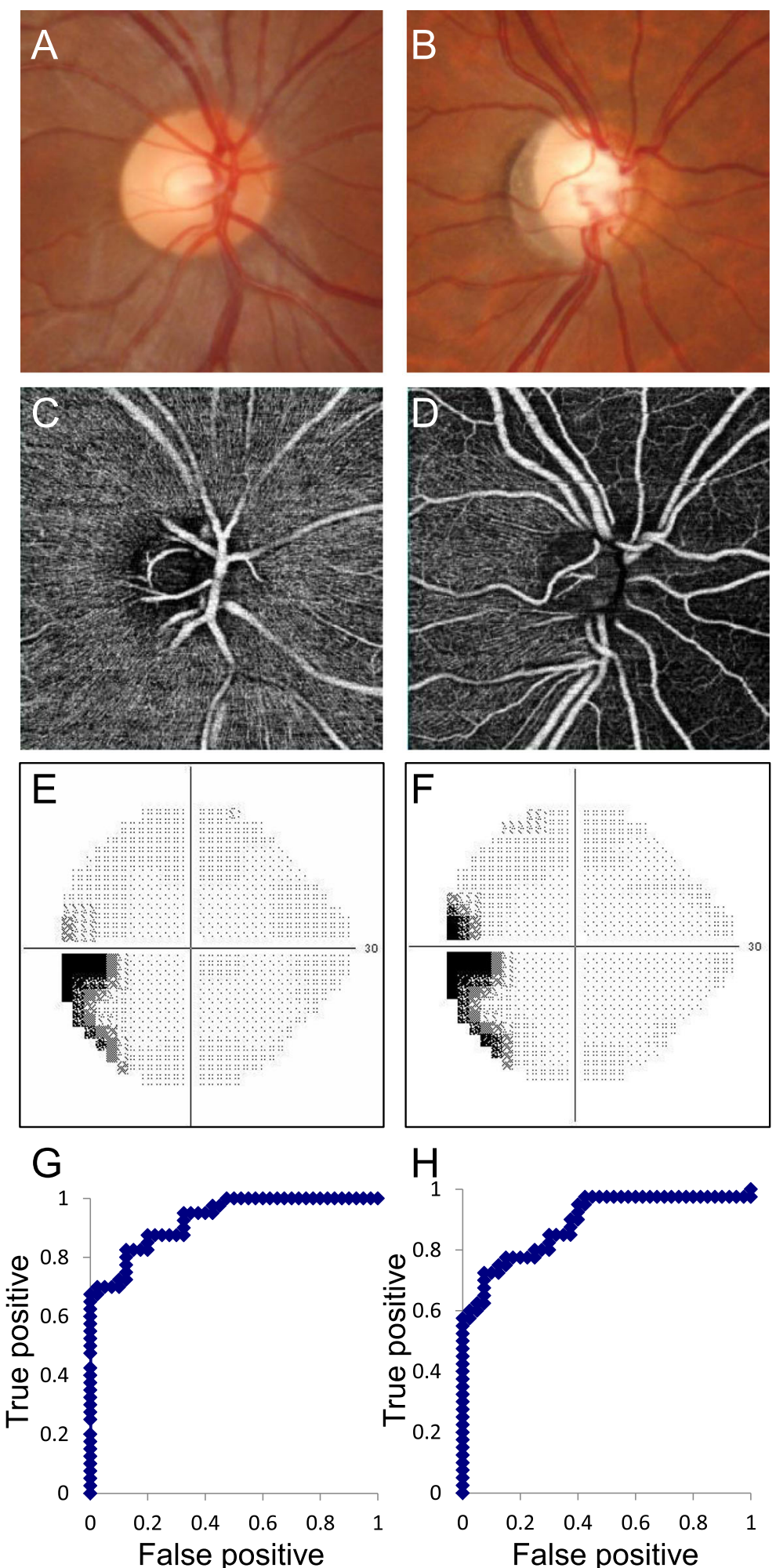

Fig. 1 Representative normal and SSOH subjects and the regional diagnostic power of RPC density for SSOH. a, b: Fundus photography. $\mathbf{c}$, d: RPC density measured with OCT-A. A, C: Normal subject. b, d: SSOH subject. e, f: Grayscale map of the visual field obtained with the Humphrey field analyzer in the $\mathrm{SSOH}$ patient (E: baseline, F 3 years later). G Receiver operating characteristic (ROC) curve for superior-quadrant $\mathrm{cpRNFLT}$. H: ROC curve for the 1 o'clock sector of cPRNFLT 
Table 1 Comparison of RPC density in normal and $\mathrm{SSOH}$ subjects

\begin{tabular}{|c|c|c|c|}
\hline & $\mathrm{RPC}(\%)$ & & \\
\hline & Normal $(n=40)$ & $\mathrm{SSOH}(n=40)$ & $p$ \\
\hline Average & $46.1 \pm 3.3$ & $39.6 \pm 4.7$ & $<0.001^{* * *}$ \\
\hline Superior & $50.3 \pm 7.1$ & $36.6 \pm 7.1$ & $<0.001^{* * *}$ \\
\hline Nasal & $37.6 \pm 5.7$ & $31.5 \pm 8.1$ & $<0.001^{* * *}$ \\
\hline Inferior & $50.7 \pm 5.8$ & $44.0 \pm 10.2$ & $<0.001^{* * *}$ \\
\hline Temporal & $45.9 \pm 5.6$ & $46.3 \pm 6.6$ & 0.756 \\
\hline Superior & & & \\
\hline 11 & $56.6 \pm 8.4$ & $49.6 \pm 10.7$ & $0.002^{* *}$ \\
\hline 12 & $43.0 \pm 11.7$ & $30.5 \pm 13.3$ & $<0.001^{* * *}$ \\
\hline 1 & $51.5 \pm 11.2$ & $30.3 \pm 12.9$ & $<0.001^{* * *}$ \\
\hline Nasal & & & \\
\hline 2 & $42.7 \pm 9.5$ & $33.6 \pm 13.7$ & $<0.001^{* * *}$ \\
\hline 3 & $31.6 \pm 8.1$ & $29.0 \pm 10.9$ & 0.238 \\
\hline 4 & $38.7 \pm 7.8$ & $32.0 \pm 11.7$ & $0.004^{* *}$ \\
\hline Inferior & & & \\
\hline 5 & $45.9 \pm 9.9$ & $36.8 \pm 12.5$ & $<0.001^{* * *}$ \\
\hline 6 & $44.9 \pm 8.5$ & $41.3 \pm 13.6$ & 0.158 \\
\hline 7 & $61.3 \pm 9.0$ & $54.9 \pm 11.3$ & $0.007^{* *}$ \\
\hline Temporal & & & \\
\hline 8 & $46.5 \pm 8.3$ & $47.2 \pm 8.6$ & 0.700 \\
\hline 9 & $38.2 \pm 7.3$ & $41.9 \pm 8.1$ & $0.039^{*}$ \\
\hline 10 & $52.7 \pm 8.9$ & $50.0 \pm 10.7$ & 0.226 \\
\hline
\end{tabular}

overall RPC density $(p=0.391$, AUC $=0.568)$ or $\mathrm{RPC}$ density in the superior quadrant $(p=0.268,0.593)$, as shown in Table 3.

\section{Discussion}

We found that the power of RPC density to differentiate $\mathrm{SSOH}$ patients and controls was strong, especially in the superior, nasal, and inferior areas. Previously, we used laser speckle flowgraphy to show that $\mathrm{SSOH}$, a congenital anomaly, causes reduced microcirculation in the $\mathrm{ONH}$ [5], that the quadrant $\mathrm{MT}$ ratios in the $\mathrm{ONH}$ have a strong power to differentiate SSOH and NTG (indicating that $\mathrm{SSOH}$ has a fundamentally different pathogenesis from NTG) [5], and that the structure of the deep layers of the $\mathrm{ONH}$, around the lamina cribrosa, are correlated to MT, but not RPC density [6]. Here, we found that the density of the peripapillary retinal microvasculature decreased in $\mathrm{SSOH}$, but that the pattern of RPC loss was distinctly different from that reported for cpRNFLT [3]. RPC density in the 9 o'clock sector, outside the temporal area, was significantly lower, but RPC density in the 3 o'clock sector was not significantly different in the $\mathrm{SSOH}$ and normal subjects. On the other hand,
Table 2 Diagnosis power of localized RPC density in normal and $\mathrm{SSOH}$ subjects

\begin{tabular}{ll}
\hline & AUC of RPC (\%) \\
\hline Average & 0.879 \\
Superior & 0.928 \\
Nasal & 0.744 \\
Inferior & 0.706 \\
Temporal & 0.520 \\
Superior & \\
11 & 0.692 \\
12 & 0.769 \\
1 & 0.896 \\
Nasal & \\
2 & 0.729 \\
3 & 0.637 \\
4 & 0.693 \\
Inferior & \\
5 & 0.709 \\
6 & 0.614 \\
7 & 0.669 \\
Temporal & \\
8 & 0.513 \\
10 & 0.628 \\
\hline & \\
\hline & \\
\hline & \\
\hline & \\
\hline & \\
\hline
\end{tabular}

cpRNFLT was not significantly different in the 9 o'clock sector (normal: $67.6 \pm 9.9$, SSOH: $70.3 \pm 19.4, p=0.431$ ), but was in the 3 o'clock sector (normal: $63.6 \pm 12.7$, SSOH: $57.8 \pm 26.9, p<0.001)$. Thus, the location of changes in RPC density and cpRNFLT is on horizontally opposite sides of the $\mathrm{ONH}$. This may be due to lower initial RPC density in the nasal $\mathrm{ONH}$, higher RPC density in the temporal $\mathrm{ONH}$, and the location of the major retinal central vessel in the superior and inferior $\mathrm{ONH}$. These findings suggest that OCT-A might have the potential to diagnose $\mathrm{SSOH}$.

Table 3 Comparison of RPC density in $\mathrm{SSOH}$ and glaucoma subjects and the diagnostic power of regional RPC density for $\mathrm{SSOH}$

\begin{tabular}{lllll}
\hline & \multicolumn{2}{l}{} & AUC \\
\cline { 2 - 4 } & SSOH $(n=27)$ & Glaucoma $(n=23)$ & $P$ value & \\
\hline Average & $57.3 \pm 11.1$ & $54.7 \pm 10.4$ & 0.391 & 0.568 \\
Superior & $56.8 \pm 15.7$ & $62.3 \pm 18.7$ & 0.268 & 0.592 \\
Nasal & $35.7 \pm 19.1$ & $46.8 \pm 15.4$ & $0.029^{*}$ & 0.688 \\
Inferior & $68.5 \pm 15.8$ & $55.8 \pm 14.9$ & $0.005^{* *}$ & 0.725 \\
Temporal & $68.7 \pm 11.0$ & $55.0 \pm 13.0$ & $<0.01^{* * *}$ & 0.781 \\
\hline
\end{tabular}


In this study, we also performed an investigation to compare $\mathrm{SSOH}$ patients and age-matched mild- and moderate-stage glaucoma patients. RPC density in the $\mathrm{SSOH}$ patients was not significantly different overall or in the superior quadrant but was significantly higher in the inferior and temporal quadrants and lower in the nasal quadrant. These differences in RPC density are understandable, because the area of the nerve fiber layer that is vulnerable to damage is different in $\mathrm{SSOH}$ and glaucoma. Our finding also shows that RPC density may be valuable for assessing damage in SSOH. Theoretically, cpRNFLT damage in $\mathrm{SSOH}$ is developmental, while damage in glaucoma is secondary. However, the diagnostic power of RPC density to differentiate $\mathrm{SSOH}$ and glaucoma was not strong, and further study is needed to determine the relationship between cpRNFLT and vasculature.

Limitations of this cross-sectional study include a small size. This study is the first to investigate changes in RPC density in SSOH. Generally, sample size calculations are hard to perform in such exploratory studies. Another limitation was the use of a specific manufacturer-dependent method for the evaluation of RPC density, even though there are currently several differing methods of calculating RPC density with devices from different companies. We excluded patients with high myopia and applied a method [8] to compensate for optical magnification of the eye, and excluded the major central retinal vessels by using image processing with a Laplacian of Gaussian filter with noise reduction. Thus, we are the first to demonstrate the potential of RPC density measurement for SSOH diagnosis.

\section{Conclusion}

We found that RPC density decreased in $\mathrm{SSOH}$, and that changes in microcirculation were indicative of $\mathrm{SSOH}$. However, it remains unclear whether these changes are congenital or secondary to retinal nerve fiber layer degeneration. In addition to cpRNFLT and the extension of the RPE over the nasal disc margin [4], OCT-A may be a new, non-invasive, objective instrument for $\mathrm{SSOH}$ diagnosis.

\section{Abbreviations \\ AUC: Area under the receiver operating characteristic curve; BF: Blood flow; cPRNFLT: Circumpapillary retinal nerve fiber layer thickness; IOP: Intraocular pressure; MT: Tissue-area mean blur rate; OCT-A: Optical coherence tomography angiography; ONH: Optic nerve head; RPC: Radial peripapillary capillary; RPE: Retinal pigment epithelium; SSOH: Superior segmental optic hypoplasia}

\section{Acknowledgements}

The authors thank Mr. Tim Hilts for editing this manuscript.

\section{Authors' contributions}

MA and KO contributed to acquisition of data, analysis and interpretation of data, and writing of the manuscript. TK contributed to analysis and interpretation of data, writing of the manuscript, and technical support. TN contributed to acquisition of funding, writing of the manuscript, and general supervision. The authors read and approved the final manuscript.

\section{Funding}

This manuscript was supported in part by a JST grant from JSPS KAKENHI Grants-in-Aid for Scientific Research (B) (T.N. 26293372), by the JST Center for Revitalization Promotion and KAKENHI Grants-in-Aid for young scientists (B) (K.O. 17 K16957) and by the Public Trust Suda Memorial Fund for Glaucoma Research. Co-author TK is employed by Topcon Corporation, a commercial company. The funders played no role in the design or conduct of the study, nor in the decision to submit the manuscript for publication.

\section{Availability of data and materials}

The datasets used and/or analysed during the current study are available from the corresponding author on reasonable request.

\section{Ethics approval and consent to participate}

This retrospective study was approved by the institutional review board of Tohoku University Graduate School of Medicine (study 2017-1-290). Written patient's informed consent was not required because the ethics committee allowed us to obtain data retrospectively from patients' records under insured medical treatment and approved to carry out by opt-out method. No patient was individually identified in this study.

\section{Consent for publication}

Not applicable.

\section{Competing interests}

The authors declare that they have no competing interests except for coauthor TK. Co-author TK is employed by Topcon Corporation, a commercial company but has no conflicts of interest associated with the content of this article.

\section{Author details}

'Department of Ophthalmology, Tohoku University Graduate School of Medicine, Sendai, Japan. ${ }^{2}$ Department of Ophthalmic Imaging and Information Analytics, Tohoku University Graduate School of Medicine, Sendai, Japan. ${ }^{3}$ R\&D Division, Topcon Corporation, Tokyo, Japan.

Received: 31 October 2019 Accepted: 27 April 2020

Published online: 24 May 2020

\section{References}

1. Iwase A, Suzuki Y, Araie M, Yamamoto T, Abe H, Shirato S, et al. The prevalence of primary open-angle glaucoma in Japanese: the Tajimi study. Ophthalmology. 2004;111(9):1641-8.

2. Yamamoto T, Sato M, Iwase A. Superior segmental optic hypoplasia found in Tajimi eye health care project participants. Jpn J Ophthalmol. 2004;48(6): 578-83.

3. Yagasaki A, Sawada A, Manabe Y, Yamamoto T. Clinical features of superior segmental optic hypoplasia: hospital-based study. Jpn J Ophthalmol. 2019; 63(1):34-9.

4. Hayashi K, Tomidokoro A, Konno S, Mayama C, Aihara M, Araie M. Evaluation of optic nerve head configurations of superior segmental optic hypoplasia by spectral-domain optical coherence tomography. $\mathrm{Br} J$ Ophthalmol. 2010;94(6):768-72.

5. Aizawa N, Kunikata H, Omodaka K, Nakazawa T. Optic disc microcirculation in superior segmental optic hypoplasia assessed with laser speckle flowgraphy. Clin Exp Ophthalmol. 2014;42(7):702-4.

6. Kiyota N, Kunikata H, Shiga Y, Omodaka K, Nakazawa T. Relationship between laser speckle flowgraphy and optical coherence tomography angiography measurements of ocular microcirculation. Graefes Arch Clin Exp Ophthalmol. 2017;255(8):1633-42.

7. Kiyota N, Kunikata H, Shiga Y, Omodaka K, Nakazawa T. Ocular microcirculation measurement with laser speckle flowgraphy and optical coherence tomography angiography in glaucoma. Acta Ophthalmol. 2018; 96(4):e485-e92

8. Iwase A, Sekine A, Suehiro J, Tanaka K, Kawasaki Y, Kawasaki R, et al. A new method of magnification correction for accurately measuring retinal vessel calibers from fundus photographs. Invest Ophthalmol Vis Sci. 2017;58(3):1858-64

\section{Publisher's Note}

Springer Nature remains neutral with regard to jurisdictional claims in published maps and institutional affiliations. 